\title{
Evaluación preoperatoria en pacientes incluidos en el programa de cooperación "Misión Milagro" en Cienfuegos
}

\section{Preoperative evaluation of patients included in "Miracle Mission" cooperation program in Cienfuegos province}

\author{
MsC. José Danilo Pacheco González,' MsC. Luis Enrique Santana Sánchez," \\ Dra. Luisa González Melian, "' Lic. María de los Ángeles González Valdés ${ }^{\text {IV }}$ \\ ' Policlínico Universitario "Cecilio Ruiz de Zárate". Cienfuegos, Cuba. \\ "Hospital Pediátrico "Paquito González Cueto". Cienfuegos, Cuba. \\ II" Policlínico Universitario "Manuel Piti Fajardo". Cienfuegos, Cuba. \\ IV Universidad de Ciencias Médicas. Cienfuegos, Cuba.
}

\section{RESUMEN}

Introducción: en la práctica médica se han creado, desde hace décadas, instrumentos que persiguen modificar y mejorar la condición previa a la cirugía. Objetivo: mejorar la evaluación preoperatoria en pacientes incluidos en el programa de cooperación "Misión Milagro."

Métodos: se realizó un estudio descriptivo retrospectivo, en el Hospital-Hotel Pasacaballos, Cienfuegos, donde se evaluaron 7956 pacientes de 12 países de América Latina y del Caribe, desde septiembre 2005 hasta abril 2007. Para la evaluación preoperatoria se propuso una metodología en cuatro etapas confeccionada en el centro. Se tomaron los siguientes datos de la base de datos en la institución: estado físico del paciente, causas del aplazamiento de la intervención, causas de cancelación por especialidades y enfermedad oftalmológica del paciente.

Resultados: predominaron los pacientes clasificados en el tipo II, 55,8 \%, según la Sociedad de Anestesia de los Estados Unidos de América. Las causas principales de aplazamiento de la cirugía fueron secreción ocular $(22,6 \%$ de los aplazados), hipertensión ocular $(19,5 \%)$, ingestión de aspirina $(17,2 \%)$ y diabetes mellitus descompensada (12,4\%). Se declararon no aptos para la intervención quirúrgica, 899 pacientes por oftalmología y 37 por medicina interna. 
Conclusiones: la metodología propuesta contribuye a mejorar la asistencia médica preoperatoria en pacientes incluidos en el programa de cooperación "Misión Milagro" en Cienfuegos.

Palabras clave: Evaluación preoperatoria, riesgo preoperatorio, ceguera, enfermedades oftalmológicas, Misión Milagro, Cienfuegos.

\section{ABSTRACT}

Introduction: for decades, instruments aimed at changing and improving the patient's condition before surgery have been created in the medical practice. Objective: to improve the preoperative evaluation of patients included in the "Miracle Mission" cooperation program.

Methods: a retrospective and descriptive study was conducted at the hotel-hospital located in Pasacaballos, Cienfuegos, in which 7956 patients from 12 Latin American and Caribbean countries were evaluated from September, 2005 to April, 2007. A customized four-phased methodology was submitted to evaluate preoperatively. The center's database provided the following pieces of information: physical status, causes of surgery postponement, causes of surgery cancellation by specialty and eye disease of the patient.

Results: type II-classified patients predominated (55.8\%, according to criteria of the US Society of Anesthesiology). The main causes of surgery postponement were ocular secretion $(22.6 \%$ of the postponed cases), ocular hypertension $(19.5 \%)$, aspirin taking (17.2\%) and decompensate diabetes mellitus (12.4\%). Eight hundred and ninety nine patients and thirty seven patients were considered noneligible for surgery due to ophthalmological and internal medicine reasons respectively.

Conclusions: The submitted methodology contributes to improving the preoperative medical care for patients included in the "Miracle Mission" cooperation program in Cienfuegos province. The achieved results suggested that it can be useful in limited financial resource settings.

Key words: Preoperative evaluation, preoperative risk, blindness, eye diseases, Miracle Mission, Cienfuegos.

\section{NTRODUCCI ÓN}

La evaluación preoperatoria es el protocolo de estudio que permite determinar el estado físico y riesgo del paciente previo al acto quirúrgico. Esta evaluación se ha ido perfeccionando con el tiempo hasta propiciar el surgimiento, desarrollo y posterior generalización de unidades especializadas en este tipo de asistencia médica.

Las enfermedades oculares constituyen un problema de salud cuya prevención merece alta prioridad. ${ }^{1,2}$ Se ha señalado que es posible evitar o curar el $80 \%$ de las discapacidades visuales, advirtiéndose que si no se ponen en marcha programas específicos, hacia el 2020 la incidencia global de la ceguera pudiera duplicarse. ${ }^{3,4}$

http://scielo.sld.cu 
En respuesta a esta situación los gobiernos de Venezuela y Cuba han desarrollado un proyecto de atención médica encaminado a la solución de las causas de ceguera prevenible en América Latina y el Caribe, al que denominaron "Misión Milagro". ${ }^{1}$ La provincia de Cienfuegos es una de las sedes elegidas para tan noble labor.

El problema identificado por la unidad preoperatoria de la sede consistía en la ausencia de un instrumento de evaluación que, de acuerdo con las características del centro, contribuyera a elevar la calidad en el servicio. En concordancia con lo expuesto, se confeccionó una guía metodológica para facilitar el cumplimiento de la actividad en esta área de trabajo.

El objetivo del estudio es mejorar la evaluación preoperatoria en los pacientes y valorar los resultados obtenidos con el procedimiento introducido en la asistencia prequirúrgica oftalmológica.

\section{MÉTODOS}

Se realizó un estudio descriptivo retrospectivo en el Hospital-Hotel Pasacaballos, Cienfuegos en el periodo transcurrido desde el 7 de septiembre de 2005 al 30 de abril de 2007, ambos inclusive. 7956 pacientes de 12 países de América Latina y del Caribe se evaluaron en el servicio preoperatorio de la institución en el periodo estudiado. Los datos primarios fueron tomados de la base de datos de la sede. Las variables incluidas fueron clasificación del estado físico, causas de aplazamiento en el servicio preoperatorio, causas de cancelación de la intervención por especialidades y enfermedad oftalmológica del paciente. Se propuso que el trabajo secuencial, según la guía metodológica introducida, comenzara por la especialidad de anestesia, continuara con medicina interna y culminara con oftalmología.

\section{Primera etapa}

La especialidad de anestesia evalúa el grado de aptitud del paciente para la intervención quirúrgica tomando en cuenta:

a) El cumplimiento de los criterios establecidos por la especialidad para la intervención propuesta.

b) La ausencia de contraindicaciones para la utilización de la anestesia establecida para este tipo de intervención.

c) El estado físico para la intervención de acuerdo con la clasificación de la Sociedad de Anestesia de los Estados Unidos de América (ASA). ${ }^{5}$

Las propuestas de la evaluación serian:

Apto: pacientes que fueran evaluados como ASA I con ausencia de limitaciones para la intervención.

No apto: pacientes con incumplimiento de los criterios establecidos para la intervención con anestesia local y aquellos con situaciones de salud que impidieran o dificultaran, por tiempo indefinido, la adecuada realización del acto quirúrgico (Ej. evaluados como ASA IV y V).

http://scielo.sld.cu 
Los pacientes clasificados como ASA II y III serían evaluados en conjunto con el clínico.

\section{Segunda etapa}

La especialidad de medicina interna evalúa el grado de aptitud del paciente para la intervención quirúrgica tomando en cuenta los resultados de la valoración de las condicionales clínicas de riesgo mayor y la presencia o no de predictores de riesgo para intervenciones no cardiacas $^{6}$ (modificado de la propuesta del Colegio de Cardiología de los Estados Unidos de América).

Condicionales no cardiacas de riesgo mayor

- Situaciones clínicas no cardiacas clasificadas ASA IV y V.

- Situaciones de salud que dificultaran, de forma indefinida, la realización exitosa del acto quirúrgico.

Predictores de riesgo mayor

- Síndrome coronario inestable o en los últimos tres meses.

- Insuficiencia cardiaca refractaria a tratamiento médico.

- Arritmias graves.

- Valvulopatías graves.

Predictores de riesgo intermedio

- Cardiopatías compensadas.

- Diabéticos compensados (glucemia en ayunas menor de $10 \mathrm{mmol} / \mathrm{L}$ ).

Predictores de riesgo menor

- Pacientes de 70 años o más.

- Hipertensión arterial no controlada.

- Enfermedad cerebrovascular en los seis meses previos.

Se consideraron propuestas de cancelación de la intervención a:

1. Pacientes con predictores de riesgo mayor.

2. Pacientes con situaciones de salud que impidieran o dificultaran, de forma indefinida, la realización exitosa del acto quirúrgico. 
Se consideraron aplazados transitoriamente a:

1. Pacientes con descompensaciones agudas de enfermedades crónicas.

2. Pacientes con enfermedades agudas que impidieran o dificultaran, a corto plazo, la realización adecuada del acto quirúrgico.

3. Pacientes con antecedentes de ingestión de medicamentos que incrementaran el riesgo de la cirugía (ácido acetil salicílico).

Se consideraron aptos aquellos que no presentaron las limitaciones de los grupos anteriores.

\section{Tercera etapa}

La especialidad de oftalmología evalúa el grado de aptitud del paciente tomando en cuenta:

a) La confirmación diagnóstica de la enfermedad oftalmológica susceptible a la intervención dentro del marco del programa de cooperación.

b) El cumplimiento de los criterios establecidos para la intervención.

c) La ausencia de limitaciones locales al momento de su evaluación establecidas para la cirugía oftalmológica (sepsis ocular, hipertensión ocular y otras).

d) El consentimiento informado del paciente en relación con el proceder quirúrgico.

Las salidas de la evaluación serian las propuestas de:

Apto: confirmación diagnóstica de la enfermedad, cumplimiento de los criterios para la intervención, ausencia de limitaciones para la ejecución y la firma del consentimiento informado por el paciente.

Aplazado: presencia de limitaciones momentáneas para la ejecución del acto quirúrgico en paciente con enfermedad oftalmológica susceptible a la intervención dentro del programa y ratifica con su firma el consentimiento informado.

No apto: el no cumplimiento de los criterios establecidos por la especialidad para la operación o el no consentimiento del paciente.

\section{Cuarta etapa}

Una vez concluido el trabajo diario se procedió por el colectivo a la ratificación de la condición de:

Aptos: a los pacientes según criterios empleados.

Aplazados: a los evaluados como tal (previo análisis y discusión). 
No aptos: para aquellos que no cumplían los requisitos para la intervención (previa argumentación y consenso).

Aquellos pacientes que resultaron aplazados por razones oftalmológicas o clínicas, una vez erradicada(s) la(s) causa(s) de su aplazamiento, y previa reevaluación de los mismos, se ratificaron como aptos por la especialidad correspondiente.

\section{RESULTADOS}

En la tabla 1 se observa predominio de los pacientes en las categorías II y I en conjunto (94\%).

Tabla 1. Evaluación del estado físico del paciente

\begin{tabular}{|c|c|c|}
\hline Clasificación de la ASA & No. & $\%$ \\
\hline ASA I & 3044 & 38,2 \\
\hline ASA II & 4431 & 55,8 \\
\hline ASA III & 439 & 5,5 \\
\hline ASA IV & 42 & 0,5 \\
\hline ASA V & 0 & 0,0 \\
\hline Total & 7956 & 100 \\
\hline
\end{tabular}

Fuente: base de datos en la institución.

Se constató que entre las causas principales de aplazamientos de las intervenciones quirúrgicas hubo superioridad de los pacientes con secreción e hipertensión ocular dentro de las causas oftalmológicas $(42,1 \%$ del total) y dentro de las causas clínicas hubo predominio de pacientes que ingirieron ácido acetil salicílico y que tuvieron descompensada su diabetes mellitus (29,6 \%) (tabla 2 ).

Tabla 2. Causas de aplazamiento en el servicio preoperatorio

\begin{tabular}{|l|r|r|}
\hline Causas de aplazamiento & No. & \multicolumn{1}{|c}{$\%$} \\
\hline Secreción ocular & 185 & 22,6 \\
\hline Hipertensión ocular & 159 & 19,5 \\
\hline Ingestión de ácido acetil salicílico & 141 & 17,2 \\
\hline Diabetes mellitus descompensada & 102 & 12,4 \\
\hline Hipertensión arterial descompensada & 79 & 9,6 \\
\hline Enfermedad respiratoria aguda & 54 & 6,6 \\
\hline Otras causas clínicas y oftalmológicas & 99 & 12,1 \\
\hline Total & 819 & 100 \\
\hline
\end{tabular}

Fuente: base de datos en la institución. 
En la tabla 3 se aprecia que 37 pacientes $(3,9 \%)$ presentaron un grupo de enfermedades clínicas que los inhabilitó para la cirugía y $899(96,1 \%)$ no reunieron los criterios para la intervención por la especialidad quirúrgica.

El pterigium y la catarata $(85,7 \%$ del total de los casos) fueron las principales enfermedades oftalmológicas atendidas en la sede (tabla 4).

Tabla 3. Distribución de pacientes no aptos según especialidades

\begin{tabular}{|l|r|r|}
\hline No aptos por medicina interna & No. & \% \\
\hline Síndromes coronarios agudos & 16 & 1,7 \\
\hline Arritmias graves & 8 & 0,9 \\
\hline Ulceras sépticas en miembros inferiores & 7 & 0,7 \\
\hline Accidentes vasculares encefálicos & 4 & 0,4 \\
\hline Demencia senil & 2 & 0,2 \\
\hline Total & 37 & 3,9 \\
\hline No aptos por oftalmología & & \\
\hline Total & 899 & 96,1 \\
\hline Total de no aptos en la sede & 936 & 100 \\
\hline
\end{tabular}

Fuente: base de datos en la institución.

Tabla 4. Enfermedades oftalmológicas atendidas en el servicio

\begin{tabular}{|l|r|r|} 
Enfermedades oftalmológicas & \multicolumn{1}{|c|}{ No. } & \multicolumn{1}{|c|}{$\%$} \\
\hline Pterigium & 3790 & 47,6 \\
\hline Catarata & 3025 & 38,1 \\
\hline Opacidad capsular posterior & 552 & 6,9 \\
\hline Otras causas & 589 & 7,4 \\
\hline Total & 7956 & 100 \\
\hline
\end{tabular}

Fuente: base de datos en la institución.

\section{DISCUSIÓN}

La valoración que precede el acto quirúrgico tiene entre sus objetivos la obtención de datos para la historia clínica, la estimación del riesgo individual, la obtención del consentimiento informado, el aporte de información sobre dieta, medicación y la preparación psicológica de cada caso para la intervención. ${ }^{7,8}$ Tal estimación permite establecer la presencia, severidad y estabilidad de algunas enfermedades que pueden comprometer el éxito de la cirugía, ${ }^{9}$ revisar el tratamiento farmacológico de los pacientes para precisar la ingestión de fármacos que pueda incrementar el riesgo operatorio (sangrado) o contraindiquen la técnica anestésica a emplear ${ }^{10,11}$ y determinar la aptitud del paciente para permanecer acostado durante la intervención. ${ }^{12,13}$ 
La clasificación $\mathrm{ASA},{ }^{5}$ desde su introducción, ha sido empleada en todos los servicios preoperatorios. Más del $90 \%$ de los casos evaluados estuvieron comprendidos en las categorías ASA I-II, lo cual era presumible dado el tipo de intervenciones que se realizan dentro del marco del programa de cooperación. La mayoría de los que presentaban pterigium fueron clasificados ASA I, generalmente pacientes de menor edad que los afectados con catarata, enfermedad más frecuente en adultos mayores y en diabéticos. La clasificación, si bien permite una aproximación inicial al estado general del paciente, para enfrentar la intervención, no contribuye a precisar el grado de riesgo del paciente y su utilización habitualmente guarda escasa correlación con las complicaciones trans y postoperatorias.

Las posposiciones del acto quirúrgico estuvieron asociadas a la ausencia de conocimientos sobre las situaciones de salud diagnosticadas, la travesía azarosa desde su sitio de procedencia hasta la sede, las transgresiones dietéticas y el abandono de medicación para el control de sus enfermedades crónicas.

El síndrome coronario agudo resultó ser la principal condición clínica de inhabilitación para el acto quirúrgico y su presentación clínica más frecuente fue sin síntomas sugestivos de isquemia coronaria y con traducción electrocardiográfica equívoca. Landesberg, ${ }^{14}$ afirma que lo habitual en los infartos perioperatorios es que se presenten sin sintomatología y con un patrón electrocardiográfico no Q. Se ha expresado que la incidencia de lesión cardiaca perioperatoria es el resultado acumulativo del estado previo del paciente, el procedimiento quirúrgico, la experiencia médica, los criterios diagnósticos de infarto miocárdico y el cuidado general de la institución en particular. ${ }^{12}$

En los registros médicos deben ser precisados manifestaciones que en ocasiones son subvaloradas, entre ellas, la tos inexplicable, edemas, palpitaciones, "mareos", fluctuaciones bruscas de la tensión arterial, síntomas relacionados con la actividad física o cambios de posición, algunas de las cuales estuvieron presentes en los enfermos. La selección no idónea de los casos enviados a la sede originó que un número significativo de ellos fueran declarados no aptos por no reunir los criterios establecidos para la intervención oftalmológica. Este resultado demostró la necesidad de una mejor evaluación diagnóstica en sus lugares de procedencia.

Las guías de valoración prequirúrgica han sido empleados desde hace décadas en la práctica médica. Múltiples autores y organizaciones han creados diferentes instrumentos que persiguen modificar, mejorar y optimizar la condición previa a la cirugía. Los índices de Goldman, Detsky, Parsonnet, Eagle, NYHA, RCRI, Asociación de Cardiología del Canadá, la ACC/AHA y las Normas de Seguridad SCARE, fueron elaborados y analizados en grandes grupos poblacionales por paneles de expertos. En opinión de Eagle y otros, ${ }^{15}$ estas guías, en general, reflejan fallas en los sistemas de puntaje y han sido desarrolladas para proveer consejería en la práctica de la Medicina Basada en la Evidencia, en parte, para reducir las demandas y como una mejora subóptima del manejo perioperatorio.

Estos índices presentan limitada especificidad y excesiva cantidad de variables que los hacen poco prácticos para nuestros intereses. Los índices de Goldman, Detsky y Parsonnet, por ejemplo, utilizan un sistema de puntaje de 9 a 16 aspectos que incluyen, en algunos ítems, situaciones clínicas poco precisas y con dudosa predictibilidad del riesgo cardiovascular. Adicionalmente, la presencia de diferentes variables con distintos rangos de valor asignado limita las posibilidades de comparación entre las escalas. 
Para el protocolo del servicio en Cienfuegos, se analizaron los lineamientos de evaluación preoperatoria para cirugía no cardiaca establecidos por la ACC/AHA. ${ }^{6}$ Esta clasificación, a diferencia de otras, pondera la severidad de la enfermedad cardiovascular y el tipo de intervención quirúrgica a realizar.

La inclusión de los condicionantes no cardiovasculares de riesgo mayor y la modificación de algunos aspectos de la escala ACC/AHA en el protocolo de la sede se justificaron por: 1. La necesidad de aplicar una estratificación del riesgo sencilla sin comprometer la calidad de trabajo del servicio, 2. El bajo riesgo quirúrgico de todas las intervenciones a realizar y 3 . El escenario de trabajo caracterizado por la atención diaria a un flujo elevado de pacientes.

La ventaja mayor del procedimiento evaluativo está en la sencillez de la metodología utilizada para las intervenciones propuestas. La ausencia de complicaciones trans y postoperatorias, imputables a la evaluación preoperatoria, corrobora la viabilidad del proceder realizado. No obstante, se requieren otras experiencias y un grado mayor de aplicación para establecer conclusiones definitivas al respecto.

Finalmente, consideramos que la propuesta metodológica resulta adecuada para la evaluación preoperatoria en la sede "Misión Milagro", Cienfuegos. Los resultados obtenidos sugieren que la aplicación del procedimiento en otras sedes del programa de cooperación pudiera ser útil, especialmente en escenarios con recursos financieros limitados.

\section{REFERENCI AS BI BLI OGRÁFICAS}

1. Jalilo SM, Martín Y, González D, Gómez N. Caracterización clínica-epidemiológica de enfermedades oculares. San Juan y Martínez. 2006-2007. Avances [Internet]. Jul-sep 2008 [citado 1 Nov 2011];10(3). Disponible en: http://www. misionmilagro.sld.cu/vol3no3/inv3306.php

2. Taylor HR, Pezzullo ML, Keeffe JE. The economic impact and cost of visual impairment in Australia. Br J Ophthalmol. 2006; 90(3):272-5.

3. Duerksen R, Limburg H, Carron JE, Foster A. Cataract blindness. Ophthalmic Epidemiol. 2006; 16:387-92.

4. Yorston D, Gichuhi S, Wood M, Foster A. Does prospective monitoring improve cataract surgery outcomes in Africa? Br J Ophthalmol. 2006; 18(2): 324-28.

5. Keats AS. The ASA classification of physical status. A recapitulation. Anaesthesiol. 1978; 49(4): 233-9.

6. Eagle KA, Berger PB, Calkins H, Chaitman BR, Ewy GA, Fleischmann KE, et al. ACC/AHA guideline update for perioperative cardiovascular evaluation for noncardiac surgery- Executive summary a report of the American College of Cardiology/American Heart Association Task Force on Practice Guidelines (Committee to Update the 1996 Guidelines on Perioperative Cardiovascular Evaluation for Noncardiac Surgery). Circulation. 2002; 105: 1257-67. 
7. Voon LW, Au Eong KG, Saw SM, Verma D, Laude A. Effect of preoperative counseling on patient fear from the visual experience during phacoemulsification under topical anesthesia: Multicenter randomized clinical trial. J Cataract Refract Surg. 2005;31(10): 1966-9.

8. Laine C, Williams SV, Wilson JF. In the clinic. Preoperative evaluation [abstract]. Ann Intern Med [Internet]. 2009 Jul [citado 1 Nov 2011];151(1). Available from: http://www.ncbi.nlm.nih.gov/pubmed/19581642

9. Henderson BA, Naveiras M, Butler N, Hertzmark E, Ferrufino-Ponce Z. Incidence and causes of ocular surgery cancellations in an ambulatory surgical center. J Cataract Refract Surg. 2006; 32(1):95-102.

10. Devereaux PJ, Goldman L, Cook DJ. Perioperative cardiac events in patients undergoing noncardiac surgery: a review of the magnitude of the problem, the pathophysiology of the events and methods to estimate and comunicate risk. CMAJ . 2005; 173:627-34.

11. Katz J, Feldman MA, Bass EB. Risks and benefits of anticoagulant and antiplatelet medication use before cataract surgery. Ophthalmol. 2003; 110: 1784-8.

12. Shamsuddin A. Ischemic Heart Disease. Anesthesiology Clinics. 2006; 24:461-85.

13. Sweitzer, B. Preoperative Medical Testing and Preparation for Ophthalmic Surgery. Ophthalmol Clin N Amer. 2006; 19: 163-77.

14. Landesberg G. The pathophysiology of perioperative myocardial infarction: Facts and perspectives. J Cardiothorac Vasc Anesth. 2003; 17:90-5.

15. Eagle KA, Brundage $B H$, Chaitman BR. Guidelines for perioperative cardiovascular evaluation for non-cardiac surgery: an abridged version of the report of the American College of Cardiology/American Heart Association Task Force on Practice Guidelines. Rev Col Anest. 2006;34:241-51.

Recibido: 8 de junio de 2011.

Aprobado: 16 de noviembre de 2011.

Danilo Pacheco González. Policlínico Universitario "Cecilio Ruiz de Zárate". Avenida 56 No. 4934 e/ 49 y 51. Cienfuegos, Cuba.

Coreo electrónico: enidsi@ucm.cfg.sld.cu 